\title{
Abnormalities of taste and smell after head trauma
}

\author{
PAUL J. SCHECHTER AND ROBERT I. HENKIN ${ }^{1}$ \\ From the Section on Neuroendocrinology, National Heart and Lung Institute, \\ National Institutes of Health, Bethesda, Maryland 20014, U.S.A.
}

SYNOPSIS Abnormalities of taste and smell were studied in 29 patients after head trauma. These abnormalities included decreased taste acuity (hypogeusia), a distortion of taste acuity (dysgeusia), decreased smell acuity (hyposmia), and a distortion of smell acuity (dysosmia). This syndrome can occur even after minimal head trauma and can begin months after the moment of injury. The patients exhibited a significant decrease in total serum zinc concentration (patients, $77 \pm 3 \mu \mathrm{g} / 100 \mathrm{ml}$, mean $\pm 1 \mathrm{SEM}$, vs controls, $99 \pm 2 \mu \mathrm{g} / 100 \mathrm{ml}, \mathrm{P}>0.001)$ and a significant increase in total serum copper concentrations $(113 \pm 4 \mu \mathrm{g} / 100 \mathrm{ml}$ vs $100 \pm 2 \mu \mathrm{g} / 100 \mathrm{ml}, \mathrm{P}<0.001)$ compared with control subjects. Symptoms of hypogeusia, dysgeusia, and dysosmia are frequent sequelae of head injury and are important to the patients and to their care after trauma.

Loss of olfactory acuity after trauma to the head is a well-recognized phenomenon and has been estimated to occur in $1.3 \%$ to $65 \%$ of all head injuries (Laemmle, 1931; Sumner, 1964). Blows to the occiput have been reported as more likely to produce loss of smell, presumably by a contrecoup effect, than blows to the forehead or other sites on the head (Sumner, 1964) and even trivial injuries have been shown to result in olfactory sensory deficits (Sumner, 1964). In addition, distortion of odours has been described after head trauma (Leigh, 1943; Sumner, 1964, 1967). Spontaneous recovery of olfactory acuity has been claimed in $8 \%$ to $39 \%$ of patients suffering from post-traumatic loss of smell (Leigh, 1943; Sumner, 1964).

On the other hand, abnormalities of taste sensation after head injury have received much less attention and have been reported to occur less frequently than do olfactory disorders. The first description of taste loss after head trauma was made by Ogle in 1870 . Since that time, additional cases of post-traumatic taste loss have been described in the literature. The incidence of this condition has been estimated to be approximately $0.4 \%$ of all head injuries, a much lower incidence than that for post-traumatic olfactory

1 Address for correspondence: Dr Robert I. Henkin, Section on Neuroendocrinology, Experimental Therapeutics Branch, National Heart and Lung Institute, Bldg 10, Room 7N262, Bethesda, Maryland 20014, U.S.A. disturbances (Mifka, 1965). Usually decreased taste acuity has been reported to be accompanied by decreased olfactory acuity. In the majority of cases return of taste acuity has been reported in the course of time, suggesting that this abnormality is more amenable to correction than post-traumatic smell loss (Sumner, 1967). No relationship between severity of head injury and the occurrence or duration of taste loss has been previously reported (Sumner, 1967).

Distorted taste sensations have been reported to accompany post-traumatic taste loss (Sumner, 1967). The incidence of distortion of either taste or smell or both in patients with post-traumatic taste loss has been estimated to be between $6 \%$ and 33\% (Leigh, 1943; Sumner, 1964, 1967).

However, the presumed diagnosis of 'anosmia' or 'ageusia' has been made in these studies on the basis of clinical subjective reports, without quantitative measurements to specify the degree or extent of the taste or smell losses.

In this paper we present data on 29 patients who experienced abnormalities of taste and smell after head trauma. Quantitative estimates of the losses of taste and smell were made. The relationship of the sensory losses to alterations in trace metal metabolism will be discussed.

\section{METHODS}

On the bases of careful evaluation of symptoms of 
approximately 400 patients with taste and smell complaints we have described alterations in taste and smell perception as follows (Henkin, 1971):

ABNORMALITIES OF TASTE Hypogeusia Decreased ability to taste salt, sweet, sour and/or bitter tastants.

Ageusia Inability to detect or recognize any tastant (operationally defined as inability to detect or recognize saturated solutions of $\mathrm{NaCl}$ or sucrose, $500 \mathrm{mM}$ $\mathrm{HCl}$, or $8 \mathrm{M}$ urea).

Dysgeusia General description of any distortion of normal taste perception.

Cacogeusia Abhorrent, obnoxious taste produced by oral introduction or mastication of food or drink. Foods most commonly eliciting this symptom are eggs, meats, poultry, fish, garlic, onions, tomatoes, coffee, chocolate, and most foods fried in oil or fat.

Phantogeusia Intermittent or persistent taste perceived in the oral cavity independent of external stimuli. This taste may be salty, sweet, sour, bitter, metallic, or indescribably obnoxious.

Heterogeusia Inappropriate consistent taste quality produced by oral introduction or mastication of food or drink. This quality is unusual and unexpected but not necessarily obnoxious-for example, all food tastes salty or sweet.

ABNORMALITIES OF SMell Hyposmia General decrease in olfactory acuity.

Anosmia Inability to detect or recognize any vapour at primary or accessory areas of olfaction (Henkin, 1967b).

Type I hyposmia Inability to detect or recognize any vapour at primary area of olfaction but maintenance of acuity at accessory areas of olfaction (Henkin, 1967b).

Type II hyposmia Decreased acuity at primary area of olfaction with maintenance of acuity at accessory areas of olfaction (Henkin, 1967b).

Dysosmia General description of any distortion of normal smell perception.

Cacosmia Abhorrent, obnoxious smell produced by the inhalation of odorants. Odorants which most commonly elicit this symptom are from perfumes, soaps, automobile exhaust, hair sprays, and from most foods which elicit cacogeusia.
Phantosmia Intermittent or persistent odour, pleasant or unpleasant, perceived when no apparent odorant is present.

Heterosmia Inappropriate smell of consistent nature associated with odorants. This smell is unusual and unexpected but not necessarily foul or obnoxious.

MEASUREMENT OF TASTE AND SMELL ACUITY Taste acuity was measured by determining detection and recognition thresholds for representatives of the salt, sweet, sour, and bitter taste qualities by a forcedchoice, three-stimulus drop technique (Henkin et al., 1963; Henkin et al., 1971). Sodium chloride was used as representative of the salt taste quality; sucrose, for sweet $\mathbf{H C l}$, for sour; and urea, for bitter. 'Detection threshold' was defined as the lowest concentration of solute consistently detected as different from water. 'Recognition threshold' was defined as the lowest concentration of solute consistently recognized correctly as salty, sweet, sour, or bitter. Taste thresholds were measured on one to four separate occasions in each subject. The lowest detection and recognition thresholds were used for analysis whenever more than one measurement was obtained.

Olfactory acuity was measured by determining detection and recognition thresholds for the vapours of pyridine in water and for nitrobenzene and thiophene in mineral oil by a forced-choice, threestimulus sniff technique (Henkin et al., 1971; Marshall and Henkin, 1971). Smell thresholds were measured on one to three separate occasions. The lowest threshold was used for analysis whenever more than one measurement was obtained.

MEASUREMENTS OF METAL METABOLISM Serum concentrations of zinc and copper and 24 hour urinary excretion of these metals were determined. Blood and urine samples were collected in metal-free tubes and plastic containers, respectively, as previously described (Meret and Henkin, 1971). Measurements of zinc and copper were carried out by atomic absorption spectrophotometry on an IL Model 153 atomic absorption spectrometer by a method described previously (Meret and Henkin, 1971).

SUBJECTS The experimental subjects were 29 patients, 17 males and 12 females, mean age 49 years (range: 22 to 76 years), 28 Caucasians and one Negro (Table 1). These patients were referred to the Taste and Smell Clinic at the Clinical Center of the National Institutes of Health by their personal physicians for evaluation of subjective complaints referable to taste and/or smell abnormalities. The mean duration of symptoms at the time these 


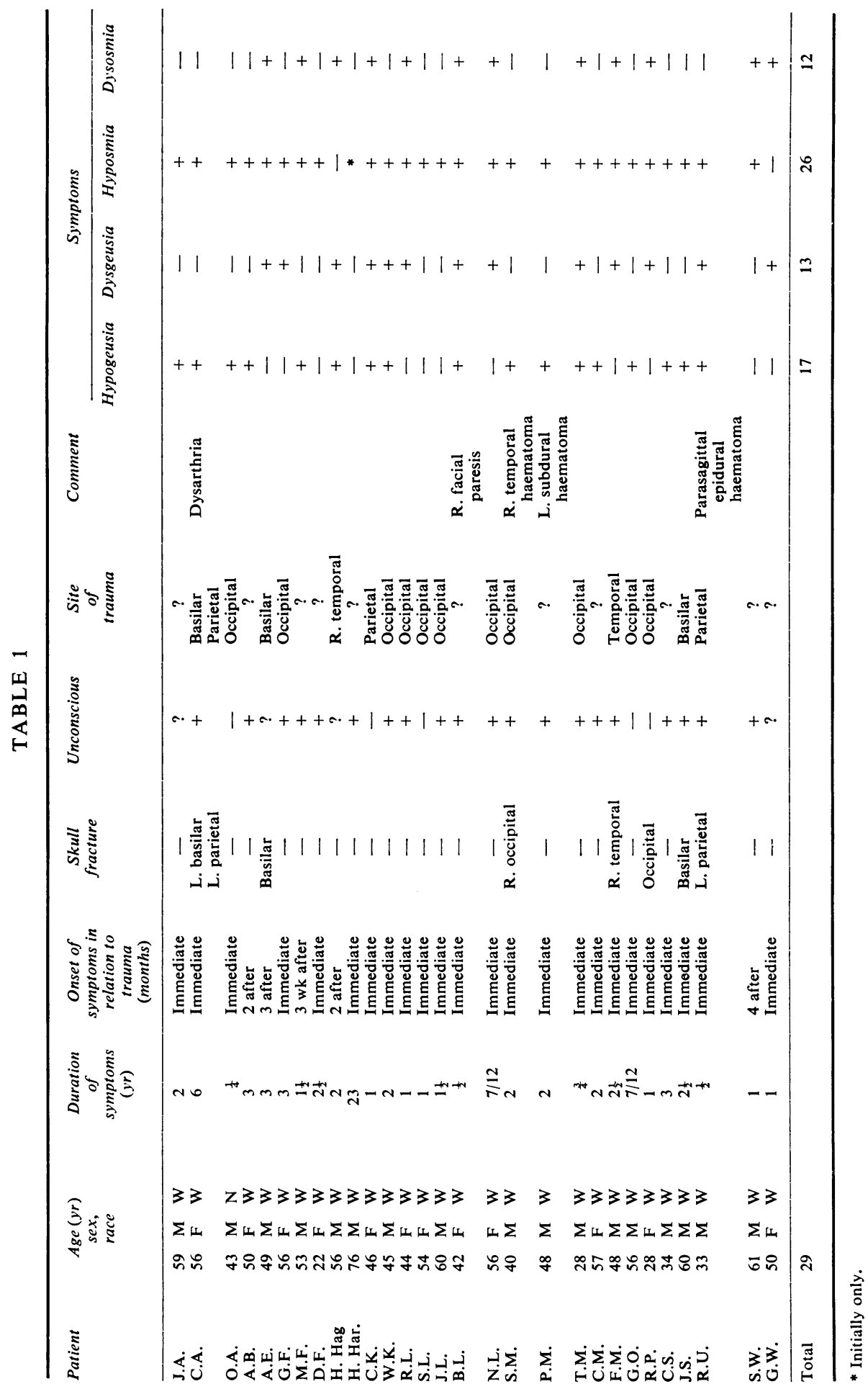


patients were first seen was $2 \frac{1}{2}$ years (range: $3 \frac{1}{2}$ months to 23 years). All patients had suffered head trauma before the onset of their symptoms and all claimed to have had normal senses of taste and smell before the trauma.

Twenty-four of the patients $(83 \%)$ noted the onset of changes in taste or smell immediately after the trauma or as soon as their mental state became clear. The other five patients noted onset of symptoms from three weeks to four months after trauma.

Seven of the 29 patients $(24 \%)$ suffered various fractures of the skull after trauma (documented by radiographs of the skull): occipital fractures (two), parietal (one), basilar (two), temporal (one), and both basilar and parietal (one). The site of trauma to the remaining 22 patients varied; however, eight of 11 who could specifically localize a site reported that trauma occurred in the occipital area. Two patients had a subdural haematoma (one right temporal region, one left temporoparietal region) which were surgically evacuated; one had an epidural haematoma in the parasagittal area.

Of the 25 patients in whom this information could be obtained, 20 reported a period of unconsciousness after trauma which lasted an extended period of time. In three patients the trauma was considered to be so trivial by the patient that medical treatment was not sought. In two cases head trauma was severe enough to produce other permanent neurological deficits.

The control subjects were 35 normal volunteers without disease and 60 patients with various diseases but without any subjective abnormality of taste or smell. These subjects were 44 Caucasian males and 51 Caucasian females, aged 15 to 77 (mean: 39) years. The patients had diseases in which zinc and copper metabolism was not altered.

EVALUATION OF PATIENTS Each patient underwent an extensive examination of the head and neck region which revealed no pathology related to the head trauma. Fungiform, circumvallate, and palatal papillae were present in each patient and bilateral parotid salivary flow rates and pharyngeal and laryngeal movements were normal. The following laboratory tests were performed in each patient: a haematological examination including haemoglobin, haematocrit, red blood cell indices, total white blood cell count with a differential count, reticulocyte and platelet counts, and erythrocyte sedimentation rate; serum sodium, potassium, chloride, carbon dioxide, calcium, phosphorus, magnesium, blood sugar, urea nitrogen, creatinine, uric acid, alkaline phosphatase, glutamic oxalacetic and glutamic pyruvic transaminases, total protein, albumin, electrophoresis and cholesterol; urin- analysis including specific gravity, $\mathrm{pH}$, evaluation of presence of glucose and bilirubin, and a microscopic examination of the sediment. Radiographs of the chest, skull, including measurement of the volume of the sella turcica (DiChiro and Nelson, 1962), and sinuses were carried out. A brain scan after intravenous injection of $99 \mathrm{~m}$ Technetium was also obtained. Results of these tests did not relate the sensory loss of any patient to any underlying pathological aetiology other than the trauma sustained.

\section{RESULTS}

SUBJECTIVE RESPONSES Subjective changes in taste and smell are shown in Table 2. Seventeen patients $(59 \%)$ complained of decreased taste acuity and $13(45 \%)$ complained of dysgeusia. Ten $(34 \%)$ noted cacogeusia, six $(21 \%)$ reported phantogeusia, and seven $(24 \%)$ experienced heterogeusia. Seven patients experienced more than one specific symptom of dysgeusia and three reported the presence of cacogeusia, phantogeusia, and heterogeusia.

TABLE 2

SUBJECTIVE ABNORMALITIES OF TASTE AND SMELL IN PATIENTS AFTER HEAD TRAUMA

\begin{tabular}{|c|c|c|c|}
\hline \multicolumn{4}{|c|}{ Taste } \\
\hline \multirow[t]{2}{*}{ Hypogeusia $(\%)$} & \multicolumn{3}{|c|}{ Dysgeusia $(\%)$} \\
\hline & Cacogeusia & Phantogeusia & Heterogeusia \\
\hline 59 & 34 & 21 & 24 \\
\hline \multicolumn{4}{|c|}{ Smell } \\
\hline \multirow[t]{2}{*}{ Hyposmia $(\%)$} & \multicolumn{3}{|c|}{ Dysosmia $(\%)$} \\
\hline & Cacosmia & Phantosmia & Heterosmia \\
\hline 90 & 38 & 14 & 17 \\
\hline
\end{tabular}

Twenty-six patients $(90 \%)$ complained of decreased olfactory acuity. One patient reported decreased olfactory acuity immediately after trauma but noted a spontaneous and gradual return to normal. The two other patients denied any decreased olfactory acuity. Twelve patients $(41 \%)$ complained of dysosmia. Of these, 11 $(38 \%)$ reported cacosmia, four $(14 \%)$ reported phantosmia, and five $(17 \%)$ reported heterosmia. Five patients experienced more than one specific 
TABLE 3

MEDIAN DETECTION AND RECOGNITION THRESHOLDS FOR FOUR TASTE QUALITIES IN 29 PATIENTS WITH HYPOGEUSIA AFTER HEAD INJURY

\begin{tabular}{lccccc}
\hline \multirow{2}{*}{ Taste quality } & \multicolumn{2}{c}{ Patients } & & \multicolumn{2}{c}{ Normal subjects } \\
\cline { 2 - 3 } \cline { 5 - 6 } & MDT/MRT & Range & & MDT/MRT & Range \\
\hline $\mathrm{NaCl}$ & $75 / 150$ & $6-650 / 30--^{*}$ & & $12 / 30$ & $6-60 / 6-60$ \\
Sucrose & $60 / 90$ & $12-800 / 30--^{*}$ & & $12 / 30$ & $6-60 / 6-60$ \\
HCl & $15 / 90$ & $0 \cdot 8->500 / 3->500$ & & $3 / 6$ & $0 \cdot 5-6 / 0 \cdot 8-6$ \\
Urea & $650 / 800$ & $90->8,000 / 90->8,000$ & & $120 / 150$ & $90-150 / 90-150$ \\
\hline
\end{tabular}

MDT: median detection threshold in $\mathrm{mM}$. MRT: median recognition threshold in $\mathrm{mM}$.

* Inability to recognize saturated solutions of $\mathrm{NaCl}$ or sucrose.

symptom of dysosmia and three reported the presence of all three.

Because of decreased palatability of food, particularly associated with dysgeusia and dysosmia, nine patients $(31 \%)$ sustained weight losses of 4 to 25 pounds ( 2 to $5 \mathrm{~kg}$ ). Of these nine, six experienced symptoms of cacogeusia and/or cacosmia. Sixteen patients $(55 \%)$ reported that they had noticeably increased the addition of $\mathrm{NaCl}$ to food to obtain the preferred salty taste and $\operatorname{six}(21 \%)$ noted that they had to increase the amount of sugar they normally added to their food or drink to produce the preferred sweet taste.

Six of the 17 patients questioned $(35 \%)$ reported a decrease in libido which was directly related in time to the trauma. Whether this related to the trauma, per se, or to the sensory loss resulting therefrom, or both is unclear. However, patients with the syndrome of idiopathic hypogeusia, without a history of trauma, often reported a similar loss of libido (Henkin et al., 1971; Schechter et al., 1972).
OBJECTIVE MEASUREMENTS Taste Median detection and median recognition thresholds for four taste qualities in the patients are compared with those of control subjects in Table 3; in the patients seven of eight threshold measurements were elevated above the upper limit of the normal range; the median detection threshold for sucrose was the only one which was within the normal range. Four patients exhibited ageusia for specific tastants; for $\mathrm{NaCl}$ (one patient), $\mathrm{HCl}$ (two patients), and urea (four patients). Nine patients exhibited elevated detection and recognition thresholds for all four tastants. Fifteen had elevated detection and recognition thresholds for sucrose; 22 for $\mathrm{NaCl} ; 27$ for $\mathrm{HCl}$; and 25 for urea.

Each patient studied exhibited elevated detection or recognition thresholds for at least one taste quality. In four patients threshold elevations were minimal, the major abnormality consisting of a confusion in the recognition of sour and bitter. These latter patients complained primarily of dysgeusia. Eleven patients who

TABLE 4

MEDIAN DETECTION AND RECOGNITION THRESHOLDS FOR THREE VAPOURS IN 28 PATIENTS WITH HYPOSMIA AFTER HEAD INJURY

\begin{tabular}{|c|c|c|c|c|}
\hline \multirow[t]{2}{*}{ Vapour } & \multicolumn{2}{|c|}{ Patients } & \multicolumn{2}{|c|}{ Normal subjects } \\
\hline & $M D T / M R T$ & Range & $M D T / M R T$ & Range \\
\hline $\begin{array}{l}\text { Pyridine } \\
\text { Nitrobenzene } \\
\text { Thiophene }\end{array}$ & $\begin{array}{c}10^{-1} / 10^{2} \\
10^{-1} / \mathbf{A} \\
\mathbf{A} / \mathbf{A}\end{array}$ & $\begin{array}{l}10^{-3}-* / 10^{-3}-^{*} \\
10^{-3}-* / 10^{-2} L^{*} \\
\left.10^{-4}{ }^{*} / 10^{-3}\right]^{*}\end{array}$ & $\begin{array}{l}10^{-7} / 10^{-3} \\
10^{-6} / 10^{-3} \\
10^{-7} / 10^{-3}\end{array}$ & $\begin{array}{l}10^{-8}-10^{-5} / 10^{-4}-10^{-2} \\
10^{-7}-10^{-5} / 10^{-4}-10^{-2} \\
10^{-8}-10^{-5} / 10^{-4}-10^{-2}\end{array}$ \\
\hline
\end{tabular}

MDT : median detection threshold in mole/1. MRT: median recognition threshold in mole/l. A : ability to detect or recognize an absolute solution.

* Inability to detect or recognize an absolute solution. 
exhibited elevations in detection and recognition thresholds were subjectively unaware of any hypogeusia, complaining only of loss of smell.

No correlations could be made between taste qualities affected, extent of taste impairment and severity of injury, presence or absence of skull fracture, locus of blow to the head, or whether a period of amnesia followed the trauma.

Smell Median detection and recognition thresholds for three vapours in 28 of the patients are shown in Table 4. Each patient exhibited elevated detection or recognition thresholds for at least one vapour, even though three patients stated that they had no loss of smell acuity at the time of study. These latter patients subjectively complained only of taste loss. All patients exhibited detection thresholds for the three vapours tested which were elevated above the upper limit of the normal range. Nine patients exhibited at least one recognition threshold within the normal range. One demonstrated anosmia. Thirteen patients demonstrated an inability to detect or recognize at least one vapour. Five patients exhibited type I hyposmia; 23 patients exhibited type II hyposmia.

As with taste acuity, no correlations could be made between extent of olfactory loss and severity of injury, presence or absence of skull fracture, locus of blow to the head, whether or not a period of unconsciousness followed the trauma, or to the variability in time after injury at which the symptoms occurred.

\section{DISCUSSION}

The present study describes alterations in taste and smell in 29 patients after head trauma. These 29 comprise the single largest reported series of patients with post-traumatic loss of taste and the only group in whom quantitative measurements of taste acuity have been determined. Although it is not possible to estimate prospectively the incidence of taste disturbances after head trauma, careful attention to the patients' complaints and quantitative taste testing revealed a greater incidence of taste abnormalities than the $0.4 \%$ previously estimated (Sumner, 1967). In addition, although taste and smell abnormalities usually occur immediately after head injury, these results emphasize that symptoms can commence several months after trauma. These observations suggest that patients should be observed at intervals subsequent to their injury in spite of the absence of other signs or symptoms of neurological dysfunction at the time of injury.

Differentiation between delayed onset of taste and smell abnormalities in patients after head injury and some forms of 'idiopathic' impairment of taste and smell can be difficult. Aetiological factors such as the sudden onset of the impairment after an influenza-like illness or some surgical procedure (Henkin et al., 1971) or the administration of a drug (Henkin, 1971a) are useful sources of information to help in this regard. The presence of acute or chronic local nasal pathology such as polyps, sinusitis, rhinitis, or nasal allergies is also useful. However, in those patients who note only a gradual, progressive diminution in acuity in taste and smell, the history of a specific incident of head trauma may be the only aetiological factor which can be used to differentiate these patients from those affected by the myriad of other causes of taste and smell abnormalities (Henkin, 1967a, b). Quantitative measurement of taste and smell loss, presence or absence of dysgeusia, and measurement of serum and urinary zinc levels may also be useful to differentiate post-traumatic from some types of 'idiopathic' impairment (Henkin et al., 1971).

No relationship between severity of head injury, presence or absence of amnesia or skull fracture, specific locus of injury, and severity of sensory loss could be made. However, the high incidence of occipital trauma in these patients suggests that a blow to this area of the skull may predispose to taste and smell alterations. Even minimal trauma may be sufficient to precipitate sensory symptoms.

In all cases olfactory symptoms invariably accompany gustatory complaints. Objective measurements of taste and smell acuity revealed that elevated smell thresholds invariably accompanied elevated taste thresholds even if patients did not complain of loss of smell.

Fifty-two percent (15 of 29) of the patients had symptoms of one or more forms of dysgeusia or dysosmia. This incidence is considerably higher than that estimated by previous investigators (Leigh, 1943; Sumner, 1964, 1967). In some patients these symptoms were much more 


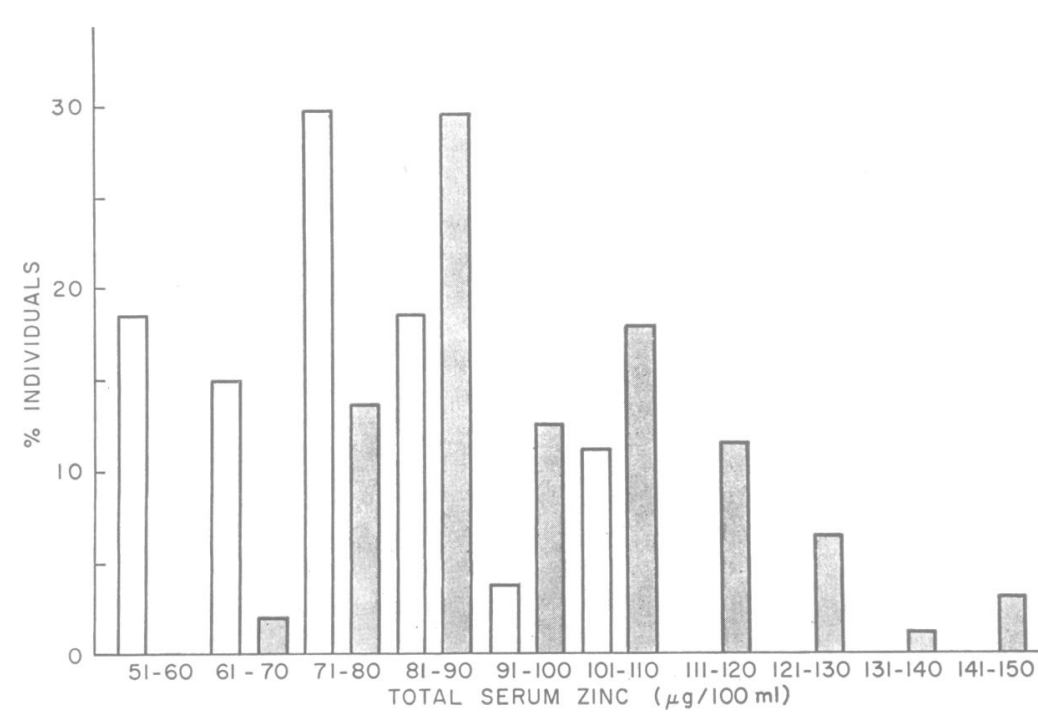

FIGURE. Total serum concentration of zinc in 27 patients with hypogeusia and hyposmia after head injury $(\square)$ and in 95 control subjects ( ). The per cent of total individuals in each category is plotted on the ordinate, serum zinc concentration in $\mu \mathrm{g} / 100 \mathrm{ml}$ on the abscissa. Note the shift of the distribution of the serum zinc concentrations of the patients toward the direction of lower than normal concentrations of zinc. disturbing than the hypogeusia or hyposmia and not uncommonly obscured the actual loss of sensory acuity. Indeed, because of the intensity of these obnoxious taste and smells some patients with dysgeusia and/or dysosmia considered their taste and smell acuity to be more acute than before their trauma.

METAL MEASUREMENTS Total serum zinc and copper concentrations and 24 hour urinary excretion of zinc and copper in the patients were compared with those from control subjects (Table 5). The patients exhibited a significant decrease in total serum zinc concentrations $(\mathrm{P}<$ 0.001 ) and a significant increase in total serum copper concentration $(\mathrm{P}<0.001)$ compared with controls. Urinary zinc excretion was also slightly but not significantly higher in the patients while no differences in the urinary excretion of copper were observed.

The Figure illustrates the differences between the distribution of concentration of total serum zinc in 95 . control subjects and in 27 patients with hypogeusia and hyposmia after head trauma. There is a shift of the distribution of serum zinc levels of the patients toward the direction of lower than normal zinc concentrations. No control subject had a total serum zinc concentration below $61 \mu \mathrm{g} / 100 \mathrm{ml}$ whereas $18 \%$ of the patients had serum zinc levels below this

\section{TABLE 5}

SERUM CONCENTRATION AND URINARY EXCRETION OF ZINC AND COPPER IN PATIENTS AFTER HEAD INJURY AND IN CONTROLS

\begin{tabular}{|c|c|c|c|c|c|c|}
\hline & \multicolumn{3}{|c|}{ Serum $(\mu \mathrm{g} / 100 \mathrm{ml})$} & \multicolumn{3}{|c|}{$\operatorname{Urinary}(\mu g / 24 h r)$} \\
\hline & $Z n^{++}$ & $\mathrm{Cu}^{++}$ & No. & $Z_{n}++$ & $\mathrm{Cu}^{++}$ & No. \\
\hline $\begin{array}{l}\text { Post- } \\
\text { trauma } \\
\text { patients }\end{array}$ & $77 \pm 3 * \dagger$ & $113 \pm 4^{*}$ & 27 & $482 \pm 54$ & $31 \pm 2$ & 22 \\
\hline Controls & $99 \pm 2$ & $100 \pm 2$ & 95 & $419 \pm 25$ & $34 \pm 3$ & 67 \\
\hline
\end{tabular}

$* P<0.001 . \quad+$ Mean \pm 1 SEm

value. On the other hand, no patient had a total serum zinc concentration greater than $110 \mu \mathrm{g} /$ $100 \mathrm{ml}$, whereas $22 \%$ of the control subjects had serum zinc levels above this concentration.

The aetiology of dysgeusia and dysosmia after head trauma is obscure. Paskind (1935) suggested that dysosmia was a symptom of nerve irritation which preceded anosmia or nerve destruction. On the other hand, Leigh (1943) suggested that dysosmia represented a stage of recovery from anosmia. Since dysosmia can commence long after the appearance of hyposmia, Paskind's hypothesis is not tenable. Leigh's suggestion also does not adequately explain the causation of these symptoms, since many cases 
of dysosmia and dysgeusia have been observed without any subjective loss of taste or smell or without objective threshold elevations of taste and smell (Schechter et al., 1972).

The magnitude of the subjective complaints of loss of smell in these patients is of interest because the majority of these patients exhibited type II hyposmia, a quantitative defect in olfactory acuity. This differs from type I hyposmia, which characteristically follows surgical interruption of the olfactory nerves, extirpation of the olfactory epithelium in maxillofacial procedures to treat carcinoma of the sinuses (Hoye et al., 1970), or after total surgical laryngectomy (Henkin et al., 1968). Many patients with type II hyposmia are unaware of their sensory loss (Henkin and Marshall, 1971). However, the suddenness of the loss of olfactory acuity which usually follows the head trauma may be the stimulus for the complaints of the patients.

The mechanism whereby a single blow to the head produces abnormalities of taste as well as smell is unclear. Post-traumatic hyposmia has been thought to be produced by a shearing of olfactory nerve filaments as they pass through the rigid cribiform plate (Ogle, 1870; Sumner, 1967). Although this hypothesis may explain the hyposmia resulting from trauma, it does not explain the accompanying abnormalities of taste, since simultaneous bilateral damage to the olfactory, lingual, glossopharyngeal, and vagus nerves does not occur in these injuries. Indeed, each patient in this study exhibited normal oral papillae, salivary flow, and pharyngeal and laryngeal function, observations which are incompatible with intact sensory function of the seventh and ninth cranial nerves. Further evidence against such a neurological lesion producing both taste and smell abnormalities is that this entire syndrome can occur after head trauma of such a mild degree that medical assistance is not sought. In addition, this syndrome occurred without any other residual neurological deficits including those of cranial nerves seven and nine in most patients studied.

It has been reported that in most cases of taste loss after head trauma there is a spontaneous return of normal taste acuity with time (Sumner, 1967). This had not occurred in any of the patients observed in this study even though intervals as long as 23 years had elapsed between the time of head injury and the time they were observed in our clinic.

We have previously demonstrated that alterations in the metabolism of trace metals either drug induced (Henkin et al., 1967; Henkin and Bradley, 1969; Henkin et al., 1972) or secondary to various disease states (Henkin and Bradley, 1969; Henkin et al., 1971; Schechter et al., 1972; Cohen et al., 1973) are associated with alterations in taste and smell acuity. Patients with the syndrome of idiopathic hypogeusia with dysgeusia, hyposmia, and dysosmia exhibit decreased concentrations of total serum zinc and increased concentrations of total serum copper (Schechter et al., 1972), the values being numerically quite similar to those observed in patients after head trauma. The oral administration of $\mathrm{Zn}^{++}$to patients with the former syndrome resulted in a decrease in symptoms and return of taste acuity towards or to normal in a significant number of patients (Schechter et al., 1972). Patients with hypogeusia after thermal injury exhibited decreased serum zinc concentrations and increased 24 hour urinary excretion of zinc (Cohen et al., 1973). Administration of the amino acid, L-histidine, associated with a significant zincuria and subsequent hypozincaemia with a loss of total body zinc produces hypogeusia, hyposmia, anorexia and, not uncommonly, dysgeusia and dysosmia (Henkin et al., 1972). Administration of $\mathrm{Zn}^{++}$, in the face of continued L-histidine administration, was associated with the rapid return of serum zinc to normal and the correction of the abnormalities of taste and smell to normal (Henkin et al., 1972). The relationship between altered sensory perception and abnormalities of zinc metabolism has been documented in various forms of liver disease (Henkin and Smith, 1971, 1972), growth retardation (Hambidge et al., 1972), malabsorptive processes of several types (Henkin, unpublished observations), and malignancies of several types (Henkin, unpublished observations).

Since patients with hypogeusia and hyposmia after head injuries exhibit serum zinc concentrations similar to those observed in patients with hypogeusia subsequent to zinc loss some aspects of the altered taste and smell perception in patients after head trauma may be secondary to alterations in zinc metabolism. If these patients were to respond to oral zinc administration in a 
similar manner to that observed in patients with idiopathic hypogeusia, then improvement in these taste and smell abnormalities might be expected. Studies are currently in progress to determine if this hypothesis be correct.

The authors wish to thank Mrs Carol J. Franklin and Mrs Diane A. Bronzert for their assistance during this study.

\section{REFERENCES}

Cohen, I. K., Schechter, P. J., and Henkin, R. I. (1973). Hypogeusia, anorexia, and altered zinc metabolism following thermal burn. Journal of the American Medical Association, 223, 914-916.

Di Chiro, G., and Nelson, K. B. (1962). The volume of the sella turcica. American Journal of Roentgenology, 87, 9891008.

Hambidge, K. M., Hambidge, C., Jacobs, M., and Baum, J. D. (1972). Low levels of zinc in hair, anorexia, poor growth, and hypogeusia in children. Pediatric Research, 6, 868-874.

Henkin, R. I. (1967). Abnormalities of taste and olfaction in patients with chromatin negative gonadal dysgenesis. Journal of Clinical Endocrinology and Metabolism, 27, 1436-1440.

Henkin, R. I. (1967a). The definition of primary and accessory areas of olfaction as the basis for a classification of decreased olfactory acuity. In Olfaction and Taste. 2, pp. 235-252. Edited by T. Hayashi. Pergamon Press: Oxford.

Henkin, R. I. (1967b). The role of taste in disease and nutrition. Borden's Review of Nutrition Research, 28, 71-87.

Henkin, R. I. (1971). Disorders of taste and smell. Journal of the American Medical Association, 218, 1946.

Henkin, R. I. (1971a). Griseofulvin and dysgeusia: implications? Annals of Internal Medicine, 74, 975-796.

Henkin, R. I., and Bradley, D. F. (1969). Regulation of taste acuity by thiols and metal ions. Proceedings of the National Academy of Science of the United States of America, 62 , 30-37.

Henkin, R. I., Gill, J. R., Jr, and Bartter, F. C. (1963). Studies on taste thresholds in normal man and in patients with adrenal cortical insufficiency: the role of adrenal cortical steroids and of serum sodium concentration. Journal of Clinical Investigation, 42, 727-735.

Henkin, R. I., Hoye, R. C., Ketcham, A. S., and Gould,
W. J. (1968). Hyposmia following laryngectomy. Lancet, 2, 479-481.

Henkin, R. I., Keiser, H. R., and Bronzert, D. (1972). Histidine-dependent zinc loss, hypogeusia, anorexia, and hyposmia. (Abstract.) Journal of Clinical Investigation, 51, 44a.

Henkin, R. I., Keiser, H. R., Jaffe, I. A., Sternlieb, I., and Scheinberg, I. H. (1967). Decreased taste sensitivity after D-penicillamine reversed by copper administration. Lancet, 2, 1268-1271.

Henkin, R. I., Schechter, P. J., Hoye, R., and Mattern, C. F. T. (1971). Idiopathic hypogeusia with dysgeusia, hyposmia, and dysosmia. A new syndrome. Journal of the American Medical Association, 217, 434-440.

Henkin, R. I., and Smith, F. R. (1971). Hyposmia in acute viral hepatitis. Lancet, 1, 823-826.

Henkin, R. I., and Smith, F. R. (1972). Zinc and copper metabolism in acute viral hepatitis. American Journal of Medical Science, 264, 401-409.

Hoye, R. C., Ketcham, A. S., and Henkin, R. I. (1970). Hyposmia after paranasal sinus exenteration or laryngectomy. American Journal of Surgery, 120, 485-491.

Laemmle, H. (1931). Ueber Geruchsstörungen und ihre klinische Bedeutung. Archiv für Ohren-, Nasen- und Kehlkopf heilkunde, 130, 22-42.

Leigh, A. D. (1943). Defects of smell after head injury. Lancet, 1, 38-40.

Marshall, J. R., and Henkin, R. I. (1971). Olfactory acuity, menstrual abnormalities, and oocyte status. Annals of Internal Medicine, 75, 207-211.

Meret, S., and Henkin, R. I. (1971). Simultaneous direct estimation by atomic absorption spectrophotometry of copper and zinc in serum, urine, and cerebrospinal fluid. Clinical Chemistry, 17, 369-373.

Mifka, P. (1965). Der traumatisch verursachte Verlust des Geruchs und Geschmackssinnes. In Late Sequelae of Head Injuries. 8th International Congress of Neurology, Vienna, 1965, Proceedings, Vol. 1, pp. 379-388. Wiener Medizinischen Akademie: Vienna.

Ogle, W. (1870). Anosmia; cases illustrating the physiology and pathology of the sense of smell. Lancet, 1, 230-231.

Paskind, H. A. (1935). Parosmia in tumorous involvement of olfactory bulbs and nerves. Archives of Neurology and Psychiatry (Chic.), 33, 835-838.

Schechter, P. J., Friedewald, W. T., Bronzert, D. A., Raff, M. S., and Henkin, R. I. (1972). Idiopathic hypogeusia: a description of the syndrome and a single-blind study with zinc sulfate. International Review of Neurobiology, Suppl. 1, 125-140.

Sumner, D. (1964). Post-traumatic anosmia. Brain, 87, 107120.

Sumner, D. (1967). Post-traumatic ageusia. Brain, 90, 187202. 